\title{
Why Should Regulators Consider Using Patient Preferences in Benefit-risk Assessment?
}

\author{
Janine A. van Til • Maarten J. IJzerman
}

Published online: 28 November 2013

(C) Springer International Publishing Switzerland 2013

\section{Why Should Regulators Consider Using Patient Preferences in Benefit-risk Assessment?}

There is an increased interest in including the patient perspective in regulatory decision making. At present, regulatory agencies such as the European Medicines Agency (EMA) in the EU and the US FDA use a combination of patient consultation and patient participation in decision committees to ensure that the patient perspective is taken into account in the decision-making process. A relatively new area of interest is the assessment of quantitative patient preferences in larger patient populations. The aim of preference elicitation is to estimate the impact and importance of known adverse effects of a new drug compared with its benefits from a patient perspective, a trade-off that is now implicitly made by the decision committee. The opportunity offered by stated preference methods is to elicit preferences in a large and representative sample of the patient population, thereby improving the reliability and validity of the preferences itself. This paper discusses the potential of patient-based preference assessment of benefits and risks in the approval process for new healthcare technologies.

\section{Drug and Device Regulation}

In the US, the Drug Safety and Risk Management Division within the FDA evaluates the safety, efficacy, and abuse

J. A. van Til $(\bowtie) \cdot$ M. J. IJzerman

Department of Health Technology and Services Research,

University of Twente, PO Box 217,

7500 AE Enschede, The Netherlands

e-mail: j.a.vantil@utwente.nl potential of drugs, and they ensure proper risk management and communication is in place. In addition, the FDA center for devices and radiological health specifically recommend devices. In Europe, the Committee for Medicinal Products for Human Use (CHMP) evaluates the risks and benefits of drugs on behalf of the EMA. In Europe, there is no centralized procedure for approval of medical devices. Regulatory authorities have to consider a wide range of different outcomes in the approval of new pharmaceuticals or medical devices. In the decision-making process, expected benefits to health must be weighed against possible safety risks, while taking into account the context of the disease that is targeted and its current management. Moreover, patient needs must be considered in judging the value of the new drug. While clinical trials provide information about the effects and adverse effects of drugs and epidemiological studies give insight into the impact of disease on the population in terms of incidence and prevalence [13], no formal instrument to assess patients' needs and the value of the new drug or device is currently used.

\section{The Patient Perspective}

In general, the perceived importance of the patient perspective in regulatory decision making is increasing. A definition of patient involvement or engagement is "involving members of the public in the agenda-setting, decision-making, and policy-forming activities of organizations/institutions responsible to policy development." [4] The first driver of patient involvement in the regulatory process is political, that is, in a democratic society patients have the right to participate in decision making. As patients are the ultimate beneficiaries of healthcare, they are considered important stakeholders in the regulatory process. 
Moreover, it is suggested that an assessment of the patient perspective would enable an evaluation of clinical, social, and ethical aspects of drug innovation that are not considered by a professional panel, which could increase the legitimacy and accountability of the regulatory decision. Lastly, but perhaps most importantly within the context of this paper, it is suggested that patient involvement could result in better alignment of regulatory decision making with the unmet needs of patients, thus increasing the quality of the decisions itself $[5,6]$.

At present, both the EMA and the FDA appoint "delegates," usually expert clinicians or other experts in healthcare to evaluate the evidence brought to the regulatory authorities and to make an appropriate value judgment in deciding upon access to the market. Deliberation takes place in multiple panel sessions, in which a qualitative evaluation of the evidence is performed. In their decision, the delegates have to take into account the validity of the studies from which the outcomes were derived, the uncertainty surrounding the data, and the context in which the drug will be used, i.e., the need for the new drug [7]. Patient involvement in regulatory decision making is operationalized differently by the EMA and the FDA; the EMA involves patients trough patient panels and consultation, while the FDA routinely includes patients or patient representatives in the decision panel.

Direct involvement of patients has its limitations. It can be questioned whether direct involvement of individual patients in panels provides sufficient democratic mandate to represent the entire patient population they represent. Moreover, it is difficult if not impossible to capture the likely variability in patient preferences in the population by asking a few individuals to provide a qualitative assessment of the impact of the disease in question and the value of benefits versus risks (harms) of the drug under review. To evaluate patient preferences in the context of their disease in a representative sample of patients, a more formal assessment of patient preferences in disease management is needed [8-10].

\section{Patient-Reported Outcomes}

Paying explicit attention to patient-reported outcomes (PROs) in the regulatory decision process is sometimes viewed as an efficient way to take into account the patient perspective [11], as PROs are almost uniformly collected in clinical trials. PROs consist of a wide range of instruments that measure different aspects of health. Most PROs are intended as a measure of benefit, not as a measure of how this benefit is valued by the patient. An exemption is quality-of-life (QoL) measurement. The main strength of QoL measurement is that it can provide a measure on the impact of disease and its management on functioning, and can link functioning to a value-based measure of health status. Accepted methodologies such as the EQ-5D, Standard Gamble, and Time Trade Off have been used over the last 20 years to measure utility for policy decision making [7]. Despite the undisputable value of QoL measurement in valuing health, they do not fit the benefit-risk framework because they value health states (and thus treatments) as a whole [12]. In addition, except for Standard Gamble, QoL measurement does not explicitly consider the risks involved in attaining that health state, a trade-off that is central to the regulatory decision-making process [13].

\section{Stated Preference Methods}

About a decade ago, stated preference methods were suggested as a method to estimate the relative importance of different aspects of health innovations from the patient perspective [14]. Although there is no consistent definition of the term "preferences," Brennan et al. [15] suggest that preferences are "statements made by individuals regarding the relative desirability of a range of health experiences, treatment options, or health states." The concept of preference assessment has strong theoretical foundations in decision theory and behavioral sciences [16].

In the regulatory context, stated preference methods could be used to identify preferences over characteristics of a drug and the trade-off between benefits and harms in choosing treatment [6]. Preference assessment in its simplest form could be obtained through ranking and/or rating benefits and harms. Ranking and rating are direct scaling methods that assign a relative importance to the value of outcomes on different attributes of a drug [17]. Although attractive because of its feasibility and low cognitive load, the lack of explicit trade-offs between benefits and harms makes ranking and rating less suitable from a methodological perspective [18]. A more elaborate way in which to collect patient preference data, which does include the trade-off that is essential to the regulatory context, is through conjoint analysis (CA). CA is a method especially tailored for the trade-off that is required in weighing benefits and risks and has strong theoretical foundations [19]. Among CA methods, the discrete choice experiment (DCE) is most often used [20]. DCEs have been shown to be methodologically valid and acceptable to patients [21, 22]. Best-worst scaling (BWS) is an adaptation of DCE that exploits the use of choice questions by asking the patient to select the best and the worst option among a set of three or more options. BWS has higher discriminating power compared with a traditional DCE, because choice data can be expanded in best-worst pairs [23]. 
Preference-based measures of value such as a DCE or BWS would allow for the estimation of the impact of benefits and risks on choice ("what would a higher risk of $\mathrm{x}$ mean for the desirability of the drug"), estimation of marginal rates of substitution ("how much risk is a patient willing to accept to receive one unit of benefit"), and estimates of how a drug is valued (relative to a comparator) by the patient. These measures of value would enable the decision committee to explicitly discuss patient preferences in disease management and to compare these with the actual benefits and harms of the drug.

\section{Patient Preferences in the Regulatory Context}

Despite the apparent appeal of stated patient preference assessment in regulatory decision making, several barriers need to be overcome to enable patient preference assessment in the practical context of benefit-risk assessment. For one, these types of studies require expertise and experience to design and analyze, and time and money to perform. In regulatory decision making, the design phase is crucial, as faulty design will result in outcomes that are not relevant in answering decision makers' questions. Moreover, from the perspective of a pharmaceutical company, the assessment of patient preferences will likely increase development costs and the time to market and might only be worthwhile if the patient perspective is thought to support a positive decision on access, not vice versa. Because the design phase can influence the outcomes of the analysis in a way that is beneficial for the drug, from the regulatory perspective the independence of preference studies must be guaranteed.

Ideally, both the value of patient preference information and the methods by which it is collected should be agreed upon by patients, agencies, and manufacturers, and it should seamlessly fit the current process of drug approval. In a "brave new world" scenario, regulatory offices might consider setting up services where pharmaceutical companies can register trials for which they feel the patient perspective is especially relevant in the decision. They would need to provide estimated benefits and risks based on trial data. Then, patients invited through patient organizations could participate in a survey to elicit preferences, and these preferences could then be fed into the decision process to inform committee members about the value of the drug to the patient. If the process of data collection, funding, and presentation of the results to decision panels is sufficiently agreed upon and organized, the consideration of unbiased and representative patient preference data could offer a novel way to consider the patient perspective in the regulatory context of drug approval.
As a first step, the potential of patient preference assessment to capture the patient perspective on the value of drugs must be recognized by regulatory agencies to the extent that they are willing to adopt it as another source of evidence that could support decisions on drug regulation. Some promising steps were made in this area. The EMA has previously acknowledged the existence of patient preference assessment through the identification and evaluation of methods for quantifying patient preferences [2] and the FDA considers evidence relating to patients' perspective as an additional source of evidence of what constitutes value in the assessment of the benefits and risks of medical devices [24].

In conclusion, while the importance of healthcare consumer involvement in medical decision making in general has been widely recognized [25], regulatory authorities have only partly met this need by including patients in decision committees and by consulting them during and informing them about the results of their appraisal process. Measures of patient preferences over the benefit and harm of new drugs collected on a larger scale in the target patient population would allow the decision committees to reflect on the need for a new technology from the patient perspective. CA or BWS could be a way to collect these data in a large and representative sample of patients in a theoretically strong manner [26]; however. many practical barriers have to be overcome to realize the full potential of stated patient preference in the regulatory context.

Acknowledgments We thank Dr. John Bridges for his input in determining the framework for this editorial, Dr. Mart oude Egbrink for his work in the analysis of current patient involvement at the EMA, and Dr. Marieke Weernink for her contributions to the part on methods for patient preference assessment and general proofreading of the manuscript. We thank the reviewer for his/her valuable feedback on the contents of this editorial. Maarten IJzerman and Janine van Til certify that they have no affiliations with or involvement in any organization or entity with any financial interest, or non-financial interest in the subject matter or materials discussed in this manuscript.

\section{References}

1. Raine J, et al. European perspective on risk management and drug safety. Clin Pharmacol Ther. 2011;89(5):650-4.

2. Abadie E et al. Benefit-risk methodology project. 2009;EMEA/ 108979/2009. http://www.ema.europa.eu/docs/en_GB/document_ library/Report/2011/07/WC500109477.pdf

3. Leong $\mathrm{J}$, et al. Is there a need for a universal benefit-risk assessment framework for medicines? Regulatory and industry perspectives. Pharmacoepidemiol Drug Saf. 2013;22(9):1004-12.

4. Rowe G, Frewer LJ. A typology of public engagement mechanisms. Science Technol Human Values. 2005;30(2):251-90.

5. Coulter A. Perspectives on health technology assessment: response from the patient's perspective. Int J Technol Assess Health Care. 2004;20(1):92-6. 
6. Bridges JF, Jones C. Patient-based health technology assessment: a vision of the future. Int $\mathrm{J}$ Technol Assess Health Care. 2007;23(1):30-5.

7. Garrison LP Jr, Towse A, Bresnahan BW. Assessing a structured, quantitative health outcomes approach to drug risk-benefit analysis. Health Aff (Millwood). 2007;26(3):684-95.

8. Parry G, Moyser G, Day N. Political participation and democracy in Britain, vol. xviii. Cambridge: Cambridge University Press; 1992. p. 509.

9. Pivik J, Rode E, Ward C. A consumer involvement model for health technology assessment in Canada. Health Policy. 2004;69(2):253-68.

10. Hansen HP, Lee A. Patient aspects and involvement in HTA: an academic perspective. Pharm Policy Law. 2011;13(3):123-8.

11. Bridges J, et al. Patient preference methods: a patient centered evaluation paradigm. ISPOR Connect. 2007;12(6):4-7.

12. Fraenkel L. Incorporating patients' preferences into medical decision making. Med Care Res Rev. 2013;70(1 Suppl):80S-93S.

13. Doward LC, McKenna SP. Defining patient-reported outcomes. Value Health. 2004;7(Suppl 1(0)):S4-8.

14. Bridges JF. Stated preference methods in health care evaluation: an emerging methodological paradigm in health economics. Appl Health Econ Health Policy. 2003;2(4):213-24.

15. Brennan DF. Patient preferences. Ann Emerg Med. 1995;26(2): 240-1.

16. Brennan PF, Strombom I. Improving health care by understanding patient preferences: the role of computer technology. J Am Med Inform Assoc. 1998;5(3):257-62.

17. Froberg DG, Kane RL. Methodology for measuring health-state preferences: II. Scaling methods. J Clin Epidemiol. 1989;42(5): $459-71$.
18. Pavlova M, Groot W, van Merode G. The importance of quality, access and price to health care consumers in Bulgaria: a selfexplicated approach. Int J Health Plann Manage. 2003;18(4): 343-61.

19. Lancaster KJ. A new approach to consumer theory. J Polit Econ. 1966;74(2):132-57.

20. Ryan M, Farrar S. Using conjoint analysis to elicit preferences for health care. BMJ. 2000;320(7248):1530-3.

21. van Til JA, et al. The use of multi-criteria decision analysis weight elicitation techniques in patients with mild cognitive impairment: a pilot study. Patient. 2008;1(2):127-35.

22. Ryan M, McIntosh E, Shackley P. Methodological issues in the application of conjoint analysis in health care. Health Econ. 1998;7(4):373-8.

23. Flynn TN. Valuing citizen and patient preferences in health: recent developments in three types of best-worst scaling. Expert Rev Pharmacoecon Outcomes Res. 2010;10(3):259-67.

24. Factors to consider when making benefit-risk determinations in medical device premarket approval and de novo classifications, D.o.H.a.H.S.F.a.D. Administration, Editor. 2012, Health and Human Services Food and Drug Administration

25. Oliver $\mathrm{S}$, et al. Involving consumers in research and development agenda setting for the NHS: developing an evidence-based approach. Health Technol Assess. 2004. 8(15):1-148, III-IV.

26. Vogt F, Schwappach DL, Bridges JF. Accounting for tastes: a German perspective on the inclusion of patient preferences in healthcare. Pharmacoeconomics. 2006;24(5):419-23. 